\section{Investigation of Mediastinitis Due to Coagulase-Negative Staphylococci After Cardiothoracic Surgery}

\author{
Maria D. Van Kerkhove, MS; Julie Parsonnet, MD; \\ Michal Weingart, $\mathrm{PhD}, \mathrm{MPH}$; \\ Lucy S. Tompkins, MD, PhD
}

Six cases of coagulase-negative staphylococcal mediastinitis were identified in the latter half of 1999. A new preoperative cleansing solution was suspected by hospital staff to be a factor in the outbreak. We evaluated this possible risk factor along with other known and suspected surgical site infection risk factors in this case-control study.

Infect Control Hosp Epidemiol 2006; 27:305-307

In the latter half of 1999, the infection control department at Stanford University Medical Center (Stanford, CA) identified 6 cases of mediastinitis caused by coagulase-negative staphylococci (CoNS). During the 4 years preceding this outbreak, the number of mediastinitis cases due to CoNS was stable (a mean of 1 case per year). Of the 6 cases of CoNS mediastinitis, Staphylococcus epidermidis was identified as the causal pathogen in 3. The pathogens responsible for the remaining 3 cases were not identified further. One case of CoNS mediastinitis occurred during each month except August, when 2 occurred. In this outbreak, all cases occurred in adult patients who underwent cardiothoracic surgery between July and November 1999. Molecular fingerprinting with pulsedfield gel electrophoresis (PFGE) ${ }^{1}$ of the 6 infecting bacterial isolates revealed that each patient was infected with a unique strain of CoNS. This suggested that the patients were not infected by a common source and that their own skin flora was the likely source of infection.

Simultaneous with the onset of the cluster of mediastinitis cases, use of a new preoperative skin cleansing solution (a combination of $62 \%$ alcohol and povidone iodine USP $5 \%$ [0.5\% available iodine $]$ ) was introduced into the cardiovascular operating rooms. This unusual outbreak of mediastinitis due to pathogens that rarely cause surgical site infections (SSIs), coupled with the initiation and cessation of use of a new preoperative cleansing solution during the same interval as the outbreak, led us to hypothesize that the cleansing solution was less effective in lowering the concentration of skin flora at the surgical site than the solution used previously and, thus, that use of the new solution may have led to bacterial regrowth during the course of surgery.

\section{METHODS}

A case of mediastinitis was defined as organ or organ-space SSI that involved the chest cavity, was caused by staphylo- cocci, occurred after a cardiothoracic surgical procedure during July-November 1999, and affected patients 30-85 years of age. ${ }^{2}$ All cases were reported to the infection control department, and in all cases, analysis of deep mediastinal tissue and/or bone revealed that CoNS bacterial pathogens were responsible for the mediastinal infections. The control group comprised a random selection of patients in the same age range who underwent cardiothoracic surgery during the study period. A case-control ratio of $1: 4$ was sought to optimize the power to detect a significant difference between the groups; however, only 22 controls met the study criteria.

A modification of the method of Pfaller $^{1}$ was used for restriction fragment analysis by PFGE. Briefly, organisms were grown overnight in broth, pelleted by centrifugation, and embedded in agarose with lysostaphin. Plugs were incubated for 1 hour at $37^{\circ} \mathrm{C}$ in lysis buffer and additional lysostaphin and then incubated for 1 hour at $55^{\circ} \mathrm{C}$ in Tris-EDTA wash buffer. Plugs were washed 5 times in wash buffer before digestion with $\mathrm{Smal}$ enzyme at $30^{\circ} \mathrm{C}$ for an interval ranging from 2 hours to overnight. Enzyme activity was stopped with EDTA, and plugs were placed into slots on a freshly poured agarose gel. Plugs were tested in duplicate or triplicate. Electrophoresis was performed with a CHEF-DR II apparatus (BioRad) at $14^{\circ} \mathrm{C}$ and $125-150 \mathrm{~mA}$ overnight. The gel was stained with ethidium bromide and photographed digitally under UV light. Relatedness among band patterns was determined by the method of Tenover et al.,

This case-control study evaluated potential risk factors for the development of mediastinitis, including age; sex; duration of preoperative preparation; prophylactic antibiotic use; duration of surgery; exposure to hospital personnel, preoperative cleansing solutions, and surgical equipment; and previous heart surgery. ${ }^{5-12}$ We were unable to assess National Nosocomial Infections Surveillance System risk class ${ }^{13}$ because 11 of the 28 subjects had missing American Society of Anesthesiologists risk scores or wound classification information. Because a large number of patients had been exposed to one surgeon, "surgeon A," the risk factor "exposure to hospital personnel" was measured as being exposed to surgeon A versus being exposed to any other surgeon at the hospital between July and November 1999. Although we suspected that use of the preoperative skin cleansing solution was a risk factor for CoNS mediastinitis and tried to account for this, the surgical report data concerning the type of preoperative cleansing solution used were found to be unreliable, and therefore we could not assess the impact of this factor.

A data collection instrument was developed to obtain information about each subject's previous medical history, laboratory procedures performed, and surgical procedures performed. The hospital's medical information system database was used to obtain missing information, if any, about cardiac catheterization procedures. Information regarding patients' general health (eg, diabetes, obesity, and immune system 
TABLE 1. Characteristics of Subjects Who Underwent Cardiothoracic Surgery at Stanford University Medical Center (Stanford, CA)

\begin{tabular}{lccc}
\hline Characteristic & $\begin{array}{c}\text { Case Subjects } \\
(n=6)\end{array}$ & $\begin{array}{c}\text { Control Subjects } \\
(n=22)\end{array}$ & $P$ \\
\hline Age, mean years & 71.7 & 65.3 & .128 \\
Male sex, \% of subjects & 83.3 & 68.2 & .64 \\
Preparation time before surgery, mean min & 73 & 71.1 & .832 \\
Contact with surgeon A, \% of subjects ${ }^{\mathrm{a}}$ & 100 & 36.4 & .016 \\
Duration of surgery, mean min & 392 & 269.7 & .038 \\
Previous heart surgery, \% of subjects & 66.7 & 9.1 & .01 \\
\hline
\end{tabular}

a See Methods for a brief description of how the values for surgeon A were calculated.

status) or lifestyle habits (eg, smoking, diet, and exercise habits) was not collected.

Data were analyzed using SAS software. ${ }^{14}$ Univariate statistical analysis by use of logistic regression for a case-control study was conducted on the variables to test the significance of each predictor on the outcome of disease. Multivariate logistic regression was used to identify a combination of risk factors associated with the odds of developing mediastinitis.

\section{RES ULT S}

A total of 6 patients (5 men and 1 woman) developed CoNS mediastinitis during the study period. The cases generally were older than the controls ( 72 vs 65 years old; $P=.13$ ) and had a greater frequency of previous heart surgery (67\% vs. $9 \%$; $P<.01$ ) (Table 1). Exposure to prophylactic antibiotics, a pulmonary artery line, a cardiac catheter, and/or a transesophageal echogram probe were similar among cases and controls. Similarly, the mean duration of time of the preoperative preparation (ie, the interval between the patient entrance into the operating room and the first incision) was nearly equivalent among cases and controls in both data sets. Perhaps the most striking differences between cases and controls was the exposure to surgeon A $(100 \%$ vs $36 \%$, respectively; $P<.05)$ and the mean duration of surgery (392 minutes [6.5 hours] vs 270 minutes [4.5 hours], respectively; $P<.05)$.

Duration of surgery (odds ratio [OR], 1.01; 95\% confidence interval $[\mathrm{CI}], 1.00-1.03)$, previous heart surgery (OR, 16.64; 95\% CI, 1.45-312.65, and exposure to surgeon A (OR, 12.15 ; $95 \%$ CI, 1.52 to $\infty$ ) were significantly associated with mediastinitis (Table 2). Multivariate analysis revealed an OR of 1.01 (95\% CI, 1.00-1.04) for duration of surgery and an OR of 3.00 ( $95 \%$ CI, 0.08 to $\infty$ ) for previous heart surgery. Because all the patients in the case group were operated on by surgeon $\mathrm{A}$, the relationship between surgery by surgeon A and mediastinitis could not be calculated.

\section{CONCLUSION}

Mediastinitis is a serious postoperative complication..$^{13}$ Molecular epidemiology findings initially indicated that this outbreak was not caused by infection from a single source and, therefore, samples were not obtained from hospital personnel for culture. Analysis of cases and controls who underwent cardiovascular surgery at the hospital between July and November 1999 revealed that increased duration of surgery-a well-documented risk factor for SSI-was associated with the risk of developing mediastinitis. This led us to look more closely at the distributions of the duration of the surgery among the cases and controls, and we hypothesized that the increased duration of surgery resulted possibly from variation in surgical technique of the surgical team. Further statistical analysis found that being operated on by surgeon A to be significantly associated with the development of the disease. Patients exposed to surgeon A tended to have longer surgery durations $(P<.01)$; however, we were not able to evaluate whether there were significant differences in certain clinically relevant characteristics between surgeon A's patients the other surgeons' patients. For example, data about the subjects' immune system status, general physical health, and preexisting conditions (such as diabetes) were not included in the analysis.

CoNS are components of the normal skin flora, and the uniqueness of each strain strongly suggests that the infection was caused by introduction of the patient's own flora during surgery. Although we could not determine the effects of preoperative skin preparation on infection, the new product had a lower concentration of the bactericidal component (povidone iodine) than did other products. Thus, its effect on the inhibition of growth of skin flora could have progressively dimin-

TAB LE 2. Univariate and Multivariate Analyses of Risk Factors for Mediastinitis After Cardiothoracic Surgery

\begin{tabular}{|c|c|c|}
\hline Statistical Analysis, Variable & $\begin{array}{c}\text { Odds Ratio } \\
\text { (95\% Confidence } \\
\text { Interval) }\end{array}$ & $P$ \\
\hline \multicolumn{3}{|l|}{ Univariate } \\
\hline Contact with surgeon $\mathrm{A}^{\mathrm{a}}$ & $12.15(1.52$ to $\infty)$ & .02 \\
\hline Duration of surgery & 1.01 (1.00 to 1.03$)$ & .01 \\
\hline Previous heart surgery & $16.64(1.45$ to 312.65$)$ & .02 \\
\hline \multicolumn{3}{|l|}{ Multivariate } \\
\hline Contact with surgeon $\mathrm{A}^{\mathrm{b}}$ & $\cdots$ & \\
\hline Duration of surgery & $1.014(1.00$ to 1.04$)$ & .10 \\
\hline Previous heart surgery & $3.00(0.08$ to $\infty)$ & .50 \\
\hline
\end{tabular}

a The median unbiased estimated is presented (the regular maximum likelihood estimate could not be calculated).

b Unable to calculate. 
ished over time. Additionally, after use of the suspected preoperative skin preparation was stopped, the CoNS infection rates among patients undergoing cardiothoracic surgery returned to baseline. A reasonable but unprovable hypothesis would be that the combination of prolonged duration of surgery with diminishing effect of the preoperative skin solution led to regrowth of the patients' CoNS during the procedure and, thus, enhanced the opportunity for contamination of the mediastinum. Cardiovascular procedures tend to have a longer duration than abdominal or orthopedic surgery procedures, for example. Thus, the decision about which preoperative cleansing solution should be used during cardiovascular surgery should consider the effectiveness of the product to reduce regrowth of skin flora over an extended period.

\section{ACKNOWLEDGMENTS}

We thank Brenda Bouvier, Tammy Schaffner, and Alexandra (Sasha) Madison, for their generous help in obtaining information about the subjects; and Ray Balise, $\mathrm{PhD}$, and Edmund Lau, MS, for their advice in statistical analysis.

Ms. Van Kerkhove and Dr. Parsonnet are from the Department of Health, Research, and Policy, and Drs. Parsonnet and Tompkins are from the Department of Medicine, Division of Infectious Diseases and Geographic Medicine, Stanford University Medical Center, Stanford, California. Ms. Van Kerkhove is from the Exponent Health Sciences Practice, New York, New York. Prof. Weingart is from Exponent Health Sciences Practice, Oakland, California. Address reprint requests to Maria D. Van Kerkhove, MS, Exponent Health Sciences Practice, 420 Lexington Avenue, Suite 1740, New York, New York 10170 (mvankerkhove@exponent.com).

Received August 4, 2004; accepted January 6, 2005; electronically published February 28, 2006.

(C) 2006 by The Society for Healthcare Epidemiology of America. All rights reserved. 0899-823X/2006/2703-0015\$15.00.

\section{REFERENCES}

1. Pfaller MA. Chromosomal restriction fragment analysis by pulsed-field gel electrophoresis—application to molecular epidemiology. In: Isenberg
HD, ed. Essential Procedures for Clinical Microbiology. ASM Press, Washington, DC; 1998:651-657.

2. Horan TC, Gaynes RP, Martone WJ, Jarvis WR, Emori TG. CDC definitions of nosocomial surgical site infections, 1992, a modification of CDC definitions of surgical wound infections. Infect Control Hosp Epidemiol 1992; 13:606-608.

3. Tenover FC, Arbeit RD, Goering RV, et al. Interpreting chromosomal DNA restriction patterns produced by pulsed-field gel electrophoresis: criteria for bacterial strain typing. J Clin Microbiol 1995; 33:2233-2239.

4. Tenover FC, Arbeit RD, Goering RV. How to select and interpret molecular strain typing methods for epidemiological studies of bacterial infections: a review for healthcare epidemiologists. The Molecular Working Group of the Society For Healthcare Epidemiology of America. Infect Control Hosp Epidemiol 1997; 18:426-439.

5. Farinas MC, Gald Peralta F, Bernal JM, Rabasa JM, Revuelta JM, Gonzalez-Mecias J. Suppurative mediastinitis after open-heart surgery: a casecontrol study covering a seven-year period in Santander, Spain. Clin Infect Dis 1995; 20:272-279.

6. Mayhall CG, ed. Hospital Epidemiology and Infection Control. 3rd ed. Baltimore, MD: Williams \& Wilkins; 2004.

7. Roy MC. Surgical site infections after coronary artery bypass graft surgery: discriminating site-specific risk factors to improve prevention efforts. Infect Control Hosp Epidemiol 1998; 19:229-233.

8. Mangram AJ, Horan TC, Pearson ML, Silver LC, Jarvis WR. Guideline for prevention of surgical site infection, 1999. Infect Control Hosp Epidemiol 1999; 20:250-278.

9. Gummert JF, Barten MJ, Hans C, et al. Mediastinitis and cardiac surgery—an updated risk factor analysis in 10,373 consecutive adult patients. Thorac Cardiovasc Surg 2002; 50:87-91.

10. Abboud CS, Wey SB, Baltar VT. Risk factors for mediastinitis after cardiac surgery. Ann Thorac Surg 2004; 77:676-683.

11. Abid Q, Nkere UU, Hasan A, et al. Mediastinitis in heart and lung transplantation: 15 years experience. Ann Thorac Surg 2003; 75:1565-1571.

12. Baldwin RT, Radovancevis B, Sweeney MS, Duncan JM, Frazier OH. Bacterial infection after heart transplantation. J Heart Lung Transplant 1992; 11:545-549.

13. NNIS System. National Nosocomial Infections Surveillance (NNIS) System Report, data summary from January 1992 through June 2003, issued August 2003. Am J Infect Control 2003; 31:481-498.

14. SAS [computer program]. Version 9.0 (TS M0). Cary, NC: SAS Institute; 2002. 